\title{
Understanding the importance of spinal injury in abusive head trauma (AHT)
}

\author{
Arabinda K. Choudhary ${ }^{1}$ (D)
}

Received: 26 August 2019 /Revised: 26 August 2019 / Accepted: 16 September 2019/Published online: 19 December 2019

(C) Springer-Verlag GmbH Germany, part of Springer Nature 2019

A growing body of evidence links abusive head trauma (AHT) to patterns of direct spinal injury such as injury to the vertebral column, ligaments and soft tissues, and to indirect evidence of injury such as spinal subdural hemorrhage in the absence of obvious spinal injury [1-3]. The article by Dr. Rabbitt et al. [4] in the current issue of Pediatric Radiology reviews the topic comprehensively and adds to the literature on abusive head trauma.

Multiple societies have recommended MR imaging of the entire spine in cases of AHT, including a recently published consensus statement on AHT supported by multiple societies [5]. But the practice of MR imaging of the spine in AHT varies across institutions, ranging from $4.3 \%$ to $84.3 \%$ [6]. The practice also varies regarding the extent of MR spinal imaging, from none, to cervical, to entire spine. The reasons for variability of spinal imaging include limited literature on the incidence of spinal findings in AHT, non-specificity of clinical signs predicting risk of spinal injury, logistical issues such as limited availability of sedation or MRI services, cost of imaging the entire spine, limited understanding of clinical and forensic markers identified on imaging, and the absence of spinal stability (which requires operative surgical management in most cases). The challenge of pathological confirmation of imaging findings, particularly of ligamentous injuries in the suboccipital region, can also be a contributory factor. These are real challenges, but so are the possible adverse outcomes for the infants and families when the workup is limited or the diagnosis incorrect. As Rabbitt et al. [4] pointed out correctly, spinal imaging findings provide valuable clinical and forensic information, and this might include suggestion of the potential mechanism of injury.

Arabinda K. Choudhary

achoudhary@uams.edu

1 Department of Radiology, University of Arkansas Medical Sciences, 4301 W. Markham, Little Rock, AR 72223, USA
So, why are spinal imaging findings important in the context of evaluation for abusive head trauma? For one, no published literature shows that spinal subdural hemorrhage is found in young infants in the absence of comminuted occipital fracture in any condition apart from abusive head trauma. This makes it a significant marker, if present, to raise concerns regarding abusive head trauma. It also helps to exclude other potential differential diagnoses that might fit with intracranial findings (such as Vitamin $\mathrm{K}$ deficiency, glutaric aciduria or Menkes syndrome) or fit with skeletal findings (such as Ehlers-Danlos syndrome or Vitamin D deficiency) but are not known to be associated with spinal subdural hemorrhage. However, in approximately 50\% of cases of AHT, spinal subdural hemorrhage is missed if only cervical spine imaging is obtained [1]. Therefore, it becomes crucial to image the entire spine in cases of suspected AHT, in the presence of intracranial subdural hemorrhage, to detect this important finding and to confidently exclude other differential diagnoses. The etiology of spinal subdural hemorrhage in AHT is still uncertain, but it is possibly reflective of the mechanism of injury in AHT. Traction on the myodural bands probably plays a significant role in shearing open the potential subdural space at the cranio-cervical junction, allowing intracranial subdural hemorrhage to migrate to spinal subdural space in abusive head trauma [1].

Second, evidence of ligamentous injuries in the suboccipital region is essentially a signature of a traumatic event [1]. This finding, if present, militates any nontraumatic differential diagnoses (such as Proteins $\mathrm{C}$ or S deficiency, or Vitamins D or K deficiency). Therefore,

- Excluding thoracolumbar spinal MR imaging in the presence of intracranial subdural hemorrhage could result in missing the clinical finding of spinal subdural hemorrhage, a significant marker for abusive head trauma; and

- Excluding both cervical and thoracolumbar spinal imaging, in addition to above, could also result in missing the clinically significant evidence of a traumatic event. 
A clinical practice of routine cervical spine MR imaging for evaluation of AHT, with the addition of thoracolumbar MR spine when intracranial subdural hemorrhage is present, is likely to provide the most comprehensive resourceconscious workup of AHT and still yield the most diagnostic result.

An important component of any research related to AHT is documenting how the index cases were identified. A consistent methodology across the published literature would help us better understand the clinical significance of imaging findings and ensure that the findings are comparable across different publications. Also, documentation of mechanism, either from confession or investigation, if available, would help us further understand and prevent these injuries. Sequential assessment of intracranial subdural hemorrhage to track development of spinal subdural hemorrhage would also help in understanding the imaging findings.

One of the challenges of the diagnosis of AHT is that, quite frequently, it is a diagnosis of exclusion and is only considered if the clinical presentation cannot be explained by natural disease processes or accidental trauma. Other authors have tried to come up with a comprehensive scoring system to test for AHT [7]. Potentially, evidence of spinal injury, including a constellation of spinal subdural hemorrhage and spinal ligamentous injury, along with evidence of intracranial injury such as spinal subdural hemorrhage and hypoxic-ischemic injury, could become a strong predictor of AHT. Further work is needed to conclude this, and the excellent article by Dr. Rabbitt et al. [4] adds to this discussion.

\section{Compliance with ethical standards}

Conflicts of interest Dr. Choudhary acts as a medico-legal expert in child abuse cases.

\section{References}

1. Choudhary AK, Ishak R, Zacharia TT, Dias MS (2014) Imaging of spinal injury in abusive head trauma: a retrospective study. Pediatr Radiol 44:1130-1140

2. Choudhary AK, Bradford RK, Dias MS et al (2012) Spinal subdural hemorrhage in abusive head trauma: a retrospective study. Radiology 262:216-223

3. Kadom N, Khademian Z, Vezina G et al (2014) Usefulness of MRI detection of cervical spine and brain injuries in the evaluation of abusive head trauma. Pediatr Radiol 44:839-848

4. Rabbitt AL, Kelly TG, Yan K et al (2019) Characteristics associated with spine injury on magnetic resonance imaging in children evaluated for abusive head trauma. Pediatr Radiol https://doi.org/10.1007/ s00247-019-0417-y

5. Choudhary AK, Servaes S, Slovis TL et al (2018) Consensus statement on abusive head trauma in infants and young children. Pediatr Radiol 48:1048-1065

6. Henry MK, Wood JN (2018) Advanced cervical spine imaging in abusive head trauma: an update on recent literature and future directions. Acad Pediatr 18:733-735

7. Hymel KP, Armijo-Garcia V, Foster R et al (2014) Validation of a clinical prediction rule for pediatric abusive head trauma. Pediatrics 134:e1537-e1544

Publisher's note Springer Nature remains neutral with regard to jurisdictional claims in published maps and institutional affiliations. 